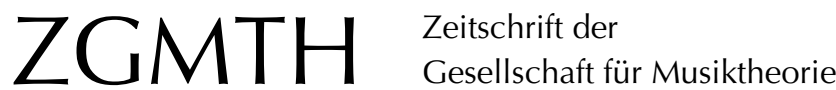

Arnecke, Jörn (2010): Dispositio, Elaboratio und Decoratio - Aspekte des Fugenthemas bei Johann Sebastian Bach. ZGMTH 7/2, 165-178. https://doi.org/10.31751/535

(C) 2010 Jörn Arnecke

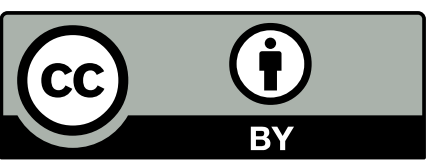

Dieser Text erscheint im Open Access und ist lizenziert unter einer Creative Commons Namensnennung 4.0 International Lizenz.

This is an open access article licensed under a

Creative Commons Attribution 4.0 International License.

veröffentlicht / first published: 05/07/2010

zuletzt geändert / last updated: 13/08/2010 


\title{
Dispositio, Elaboratio und Decoratio - Aspekte des Fugenthemas bei Johann Sebastian Bach
}

\author{
Jörn Arnecke
}

\begin{abstract}
Rekonstruiert man einen hypothetischen Entstehungsprozess Bachscher Fugenthemen entlang der von Johann Mattheson genannten Arbeitsschritte 'Dispositio<, 'Elaboratio und 'Decoratio<, so gewinnt man sowohl konkrete Erkenntnisse über das jeweilige Fugenthema als auch generelle Einsichten in die Denkweisen, die der Themenfindung und der Fugenkomposition zugrunde liegen: Das Thema lässt sich als Extrakt eines mehrstimmigen, modellbasierten Satzes verstehen, und ihm wohnt ein kontrapunktisches bzw. kombinatorisches Potential inne. Auf dieses Potential hin kann das Thema entworfen sein, und die kontrapunktischen Möglichkeiten können im Laufe der Komposition offen gelegt und entfaltet werden. Dies wird anhand der ersten vier Fugenthemen des Wohltemperierten Klaviers (1722) gezeigt. Dabei erweist sich der dreistimmige Satz strukturell als so maßgebend, dass das Ende einer dreistimmigen Exposition als der eigentliche Ausgangspunkt der Komposition erscheint. In der Unterrichtspraxis sollten diese Einsichten dazu führen, dass Themen - auch improvisatorisch - entwickelt und auf ihre kontrapunktischen Möglichkeiten hin befragt werden.
\end{abstract}

2008 widmete sich eine Doppelausgabe der ZGMTH der `Fuge dass ihre Relevanz für den Musiktheorie-Unterricht zu schwinden scheine. Durch den vorliegenden Beitrag soll die dort angestoßene Diskussion fortgeführt und stärker auf die Lehre zugespitzt werden. Hierfür wird die Betrachtung bewusst auf das Fugenthema konzentriert; ${ }^{1}$ die aufgestellten Thesen werden beispielhaft an den ersten vier Fugen des Wohltemperierten Klaviers von Johann Sebastian Bach (1722) hergeleitet und belegt. Damit wird der Weg weiterverfolgt ${ }^{2}$, diesen nicht zuletzt als Lehrwerk konzipierten Zyklus in seiner Bedeutung und pädagogischen Motivation zu entschlüsseln. ${ }^{3}$ Abschließend werden aus den Thesen Konsequenzen für die Unterrichtspraxis gezogen.

1 Der Autor knüpft damit an Überlegungen an, die er in einer Analyse des dis-Moll-Fugenthemas aus dem Wohltemperierten Klavier dargelegt hat (Arnecke 2002).

2 Auf die überaus umfangreiche Literatur zum Wohltemperierten Klavier kann im Rahmen dieser Untersuchung nur am Rande eingegangen werden; bewusst sind die Literaturbezüge auf die jüngst in der ZGMTH erschienenen Beiträge konzentriert.

3 Vgl. Deppert 2008, 288, der sich in diesem Punkt auf Hinrichsen 2008 und Schmoll-Barthel 2008 bezieht. 
Der Blick soll dabei auf die nachschöpferische Arbeit gelenkt werden. Eine Analyse hat nach herkömmlichem Verständnis die fertige Komposition zum Gegenstand. Sie zerlegt das Werk in Bestandteile oder arbeitet mittel- und hintergründige Schichten heraus; sie kann den formalen Ablauf verdeutlichen, auf Modelle verweisen, harmonische oder melodische Strukturen aufdecken ${ }^{4}$, Höreindrücke reflektieren oder Bezüge zu außermusikalischen Feldern herstellen. ${ }^{5}$ Wenn Musiktheorie jedoch den Anspruch erhebt, nachschöpferisch tätig zu sein, müsste ihr Ideal darin bestehen, musikalische Werke gewissermaßen nachzukomponieren und dadurch den Prozess von der Idee bis zur Ausarbeitung hypothetisch zu rekonstruieren. ${ }^{6}$

Die zentrale Rolle des Fugenthemas für die Konzeption einer Fuge dürfte unstrittig sein, wohl aber unterscheiden sich die Auffassungen davon, wie darauf im Unterricht Bezug genommen werden soll. Hermann Grabner schreibt in seiner Anleitung zur Fugenkomposition (1934):

`Rezepte zur Anfertigung von Fugenthemen zu geben [...], ist sinnlos, da die Erfindung eines guten Themas immer Sache der künstlerischen Inspiration sein wird. Der kompositorisch minder Begabte möge sich daher für die folgenden Übungen lieber eines der im Anhang angegebenen Themen bedienen, als sich mit einem stümperhaften selbsterfundenen Thema abzuplagen. ${ }^{7}$

Wenn aber der Unterricht zu einer nachschöpferischen Arbeit werden soll, wenn es darum geht, den Kompositionsprozess nachzuvollziehen, dann muss die These gegensätzlich lauten:

\section{These 1}

Der Bau des Themas ist die erste, grundlegende Arbeit des Fugenkomponisten.

Und dies lässt sich noch erweitern:

\section{These 2}

Aus der Grundidee der Fuge kann die Konzeption des Themas erwachsen: Engführungen, Umkehrungen usw. können in ihm angelegt werden. ${ }^{8}$

4 Vgl. Polth 2008.

5 Vgl. Jeßulat 2008.

6 Natürlich ist es unmöglich anzugeben, wie Bach vorgegangen ist und in welchen Schritten er eine Fuge konzipierte. Aber der Versuch eines Nachkomponierens lässt sowohl die Bedingungen der Entstehung als auch die konkreten kompositorischen Problemstellungen deutlicher hervortreten und ermöglicht es, Hypothesen über Bachs Kompositionsweise abzuleiten.

7 Grabner 1934, 8. Dass Grabner mit dieser Meinung nicht allein steht, zeigt sich in der fast gleich lautenden Formulierung fast fünfzig Jahre zuvor bei Ernst Friedrich Richter, der die Themenfindung als »Sache des Talents und der Inspiration« bezeichnet (1886, 54); vgl. hierzu Froebe 2008b, 243.

8 Für die Umkehrung hat auch Heinrich Deppert diese Verbindung hergestellt, bezogen auf Bachs Praeludium und Fuge in A (BWV 896): "Das Thema muss wohl für diesen Zweck entsprechend entworfen worden sein!« $(2008,295)$ Diese Vermutung führt er nicht weiter aus. 
Anders als bei Instrumentalfugen aus der Zeit um $1700^{9}$ ist bei Johann Sebastian Bach das Modell oft nicht unmittelbar erkennbar ${ }^{10}$; wir müssen also den Weg vom Modell zum Thema in mehreren Schritten zurücklegen. Dabei hilft uns eine Aussage von Johann Mattheson:

Wenn nun hier eine fernere lehrreiche Betrachtung von der Erfindungs-Kunst angestellet werden soll, so wird zuvördest nöthig seyn darzuthun, daß dieselbe Kunst drey unzertrennliche Gefährten haben müsse, ohne welche auch die allerschönsten Einfälle von schlechter Würde sind. Diese drey heissen: Dispositio, Elaboratio \& Decoratio $[\ldots]^{11}$

Wir werden also von einem elementaren Stimmführungsphänomen ausgehen, dieses ausarbeiten und schließlich diminuieren und so zu einem Thema profilieren. ${ }^{12}$

\section{Das Thema aus einer einstimmigen Linie}

Als Beispiel diene das Vorhaben, eine Fuge mit vielen Engführungen zu komponieren. Engführungen gelten in der historischen Fugenlehre als fester Bestandteil, als Krönung einer Komposition. ${ }^{13}$ So überrascht es nicht, dass ein Lehrwerk wie das Wohltemperierte Klavier diese Kunst gleich in der ersten Fuge mustergültig und in besonderer Dichte vorführt. Gesucht ist also ein Gerüstsatz, der in besonderer Weise Engführungen ermöglicht.

Hierfür schöpft der Komponist aus einem Vorrat an Möglichkeiten. Wie sich zeigen wird, lassen sich aus dem folgenden Stimmverlauf (Beispiel 1) Engführungen gewinnen:

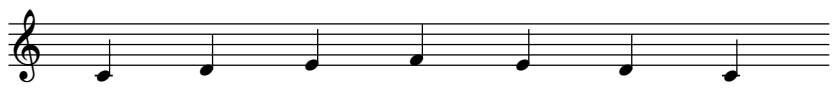

Beispiel 1: Einstimmiger Elementarvorgang, der Engführungen ermöglicht

Denn die Bogenform des Sekundgangs erlaubt es, eine Unterstimme in der Unterquart so zu führen, dass mit dem melodischen Wendepunkt von einem steigenden Sextenparallelismus in einen fallenden Terzenparallelismus gewechselt wird; sowohl Terzen als auch Sexten erlauben den doppelten Kontrapunkt in der Oktave, so dass die Konstellation auch mit vertauschter Ober- und Unterstimme möglich ist. Für diese zweistimmige Fassung bedarf es einer kleinen rhythmischen Korrektur (Beispiel 2): ${ }^{14}$

9 Vgl. Froebe 2008b, 218ff.; Polth 2008, $260 \mathrm{ff}$.

10 Eine Ausnahme bildet, wie unten ausgeführt wird, die Cis-Dur-Fuge aus dem Wohltemperierten Klavier.

11 Mattheson 1739, 122.

12 Diese Vorgehensweise beruft sich auf den Ansatz von Volkhardt Preuß (2002).

13 Froebe führt hierfür Johann Gottfried Walther, Johann Mattheson, Friedrich Wilhelm Marpurg und Johann Georg Albrechtsberger an (Froebe 2008b, 228). 


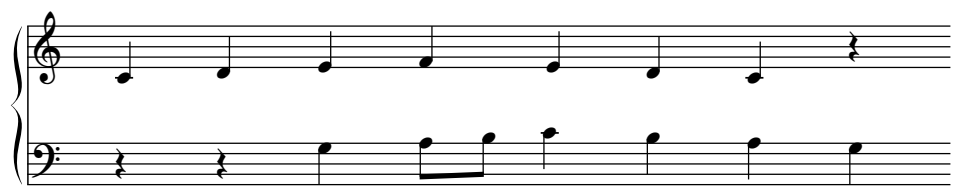

Beispiel 2: Zweistimmiger Elementarvorgang

Die rhythmische Korrektur muss in die Oberstimme übertragen werden. Zugleich ermöglicht dies, für einen besseren musikalischen Fluss die Anfangsnote zu verkürzen und mit einer Pause anzusetzen (Beispiel 3):

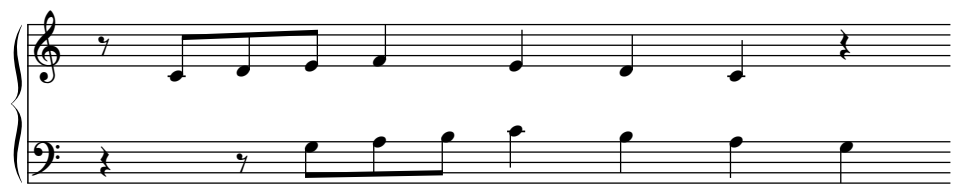

Beispiel 3: Engführung mit rhythmischer Veränderung des ursprünglichen Sekundgangs

Die terzparallele Linienführung im zweiten Teil des Beispiels legt eine Formulierung als Fauxbourdonsatz nahe ${ }^{15}$, zumal hierdurch die Möglichkeit des doppelten Kontrapunktes in der Oktave erhalten bleibt (Beispiel 4):

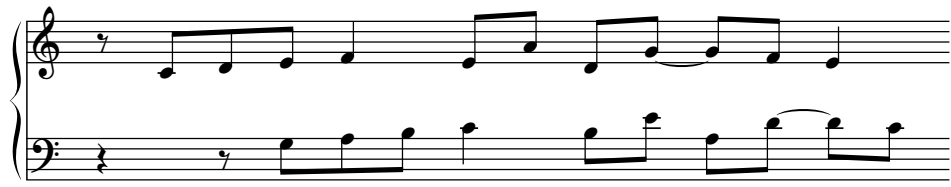

Beispiel 4: Interpretation des zweiten Abschnittes als diminuierter Fauxbourdonsatz

Wir haben also einen elementaren Stimmführungsvorgang gewählt (`Dispositio $)$ und ausgearbeitet (`Elaboratio $<$ ). Nach der Anweisung Matthesons fehlt nun noch die `Decoratio : Das Stimmgerüst soll diminuiert bzw. ornamentiert werden, als Engführung entsprechend in beiden Stimmen. Hierzu eignet sich besonders die Viertelnote, da sie der längste Notenwert und zugleich der melodische Wendepunkt ist (Beispiel 5).

14 Derartige Elementarvorgänge fasst Mauritius Vogt unter den Begriff der sphantasia simplexı. Diese bestimmt Froebe als »ein elementares, im Kern zweistimmiges und insofern für weitreichende Transformationen und Kombinationen offenes kontrapunktisches Modell« (2008b, 212). Im vorliegenden Beispiel wäre demnach eine aufsteigende mit einer absteigenden sphantasia k kombiniert.

15 Dies geht auf den Grundsatz zurück, das Thema in einen mehrstimmigen Satz einzufügen, so »daß man sich bey Erfindung desselben sogleich den $\mathrm{Baß}$ und die übrigen Gegenstimmen vorstellet" (Marpurg 1753/54, I, § 5, 29); vgl. hierzu auch Froebe 2008b, $227 \mathrm{ff}$. 


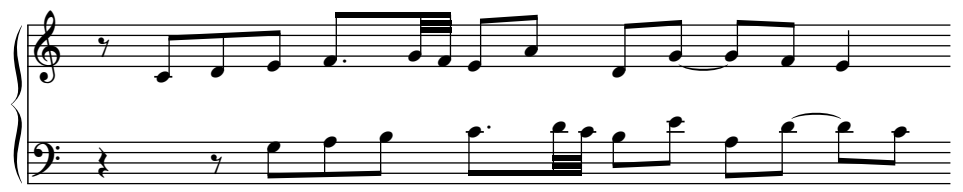

Beispiel 5: Ornamentation des melodischen Wendepunkts ${ }^{16}$

Für eine bessere Fortsetzung kann auch die Themenkadenz diminuiert werden, und so ergibt sich schlüssig die erste Engführung in Johann Sebastian Bachs C-Dur-Fuge aus dem Wohltemperierten Klavier (Beispiel 6). ${ }^{17}$

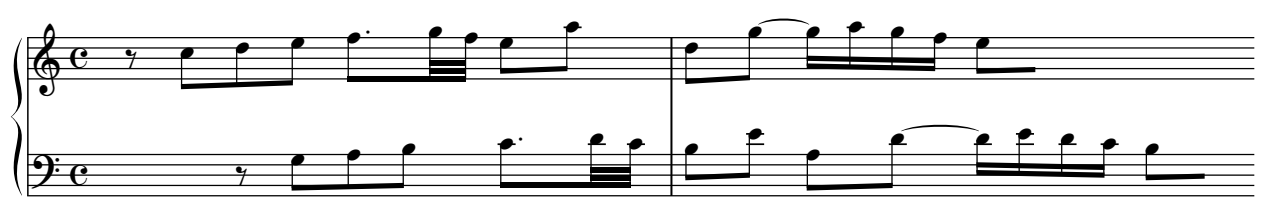

Beispiel 6: Johann Sebastian Bach, Wohltemperiertes Klavier (1722), Fuge C-Dur BWV 846, T. 7-8, Sopran und Tenor

\section{Das Thema aus einem linearen Parallelismus}

Grundlage für diese Überlegungen war ein einstimmiger Melodiebogen, aus dessen dichter Imitation die Überführung eines Sexten- in einen Terzenparallelismus resultierte. Im Folgenden soll ein linearer Parallelismus von vornherein als Ausgangspunkt der Themenbildung dienen (Beispiel 7).

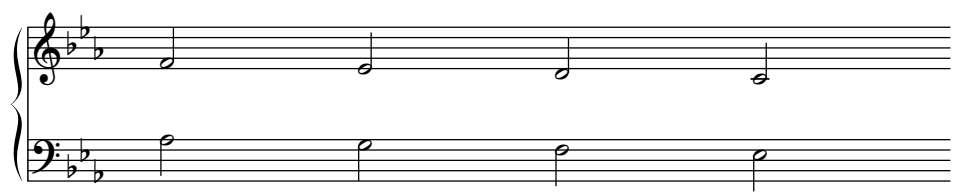

Beispiel 7: Parallele Sexten als Elementarvorgang für ein Fugenthema

Zum Sextenparallelismus lässt sich, ebenfalls in Sekundschritten verlaufend, eine Gegenbewegung bilden. Zu dieser neuen Stimme ist außerdem eine terzparallele Linie möglich, welche die vorherige Oberstimme ersetzen kann, so dass sich ein neues dreistimmiges Modell ergibt (Beispiel 8).

16 Der hier vorliegende ,vokale< Thementypus ist gegenüber der metrischen Verschiebung, die aus der engen Imitation im Abstand einer Viertel resultiert, unempfindlich. Um die metrische Indifferenz beider Einsätze zu verdeutlichen, wurden die Taktstriche weggelassen.

17 Eine andere Deutungsmöglichkeit der Themenkadenz liegt in der Verschränkung von Tenor- und Altklausel, vgl. hierzu den Abschnitt »Das Thema aus einer Klausel-Wendung». 


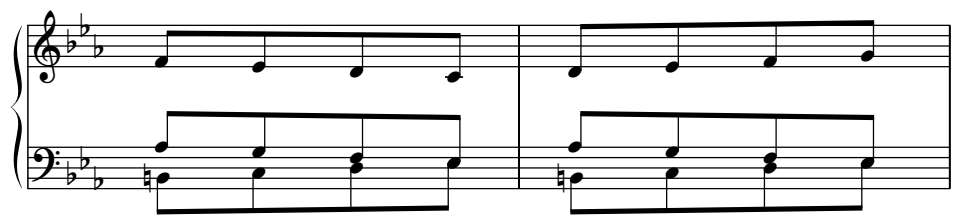

Beispiel 8: Dreistimmige Modelle mit Sext- bzw. Terzparallelen und Gegenbewegung

Beide Erscheinungen lassen sich miteinander kombinieren - die Sextparallelen stehen auf den Stationen der halben Noten, die dreistimmigen Modelle sind zwischengeschaltet bzw. fungieren als Diminutionen des einfachen Sextenparallelismus (Beispiel 9).

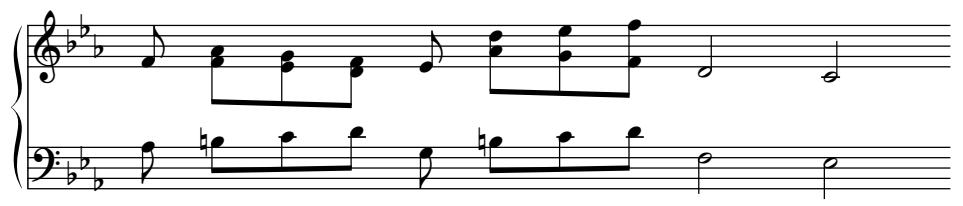

Beispiel 9: Integration der gegenläufigen Sekundgänge in den übergeordneten Sextenparallelismus

Diese gegenläufigen, dreistimmigen Modelle sind im mehrfachen Kontrapunkt der Oktave vertauschbar, z. B. können sich im ersten Modell Ober- und Unterstimme gegenseitig ersetzen. Setzt man die auf diese Weise gewonnene Stimmkombination an der Position der dritten halben Note ein, so ergibt sich folgende Themenkadenz (Beispiel 10):

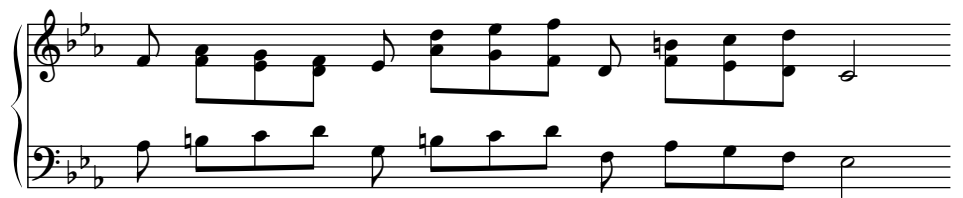

Beispiel 10: Integration dreier gegenläufiger Modelle

Nun muss wiederum die Decoratio erfolgen: Eine Stimme wird thematisch profiliert und der Satz dadurch hierarchisch organisiert ${ }^{18}$ (Beispiel 11).

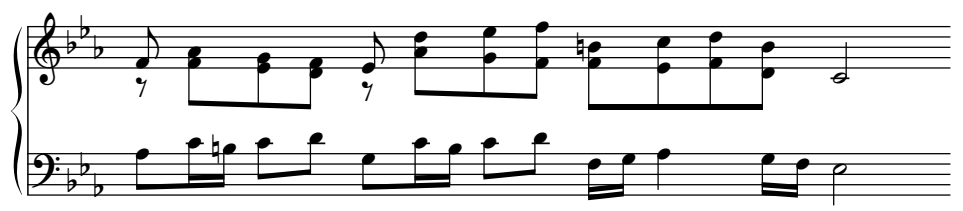

Beispiel 11: Thematisch profilierte Bassstimme im dreistimmigen Satz 
Daraus ergibt sich unmittelbar der dreistimmige Satz für den Schluss der Exposition (Beispiel 12).

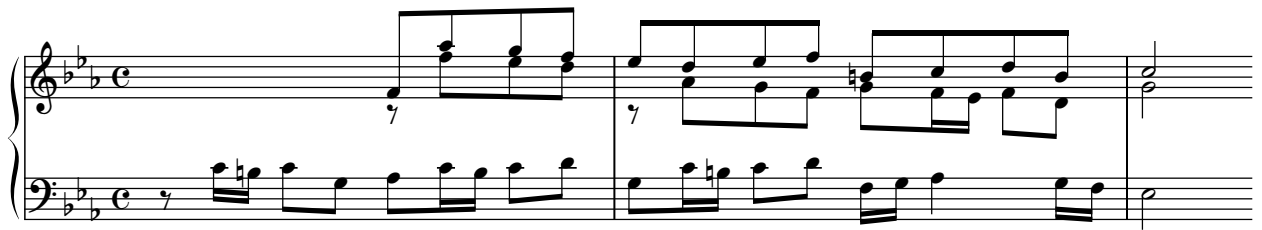

Beispiel 12: Johann Sebastian Bach, Wohltemperiertes Klavier (1722), Fuge c-Moll BWV 847, T. 7-9

\section{Das Thema aus einer Vorhaltskette}

Ein weiteres Beispiel gewinnen wir, wenn wir den einfachen Parallelismus in eine Vorhaltskette überführen, etwa den Sextenparallelismus in die 7-6-Konsekutive (Beispiel 13).

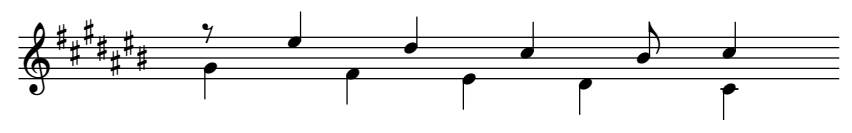

Beispiel 13: 7-6-Konsekutive

Es ist unmittelbar erkennbar, dass das Thema der Cis-Dur-Fuge auf dieser Vorhaltskette beruht und zwischen beiden Stimmen der Vorhaltskette hin und her springt. Bach baut jedoch eine metrische Beschleunigung in das Thema ein: Am Anfang ist eine Achtelnote des Modells auf drei Achtelnoten im Thema gedehnt, dann sind es zwei Achtelnoten, und schließlich stellt sich die rhythmisch regelmäßige 7-6-Folge mit Wechsel auf jeder Achtelnote ein, so dass die beiden Stimmen des Modells in der Kadenz Tenor- und Diskantklausel bilden.

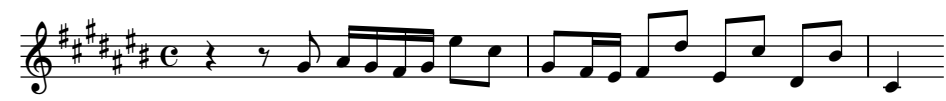

Beispiel 14: Johann Sebastian Bach, Wohltemperiertes Klavier (1722), Fuge Cis-Dur BWV 848, Thema

Im mehrstimmigen Satz lässt sich der zweite Takt des Themas klar sequenziell fassen: Wird das Thema in den Bass gesetzt, können Sextparallelen in der Oberstimme die strukturelle Oberstimme des Themas verdoppeln (Beispiel 15).

18 Die Frage der Hierarchie der Stimmen in der Fuge diskutiert Michael Polth aus dem Blickwinkel der Schenkerian Analysis (2008, 250) in Auseinandersetzung mit Carl Dahlhaus' Sichtweise (1989). 


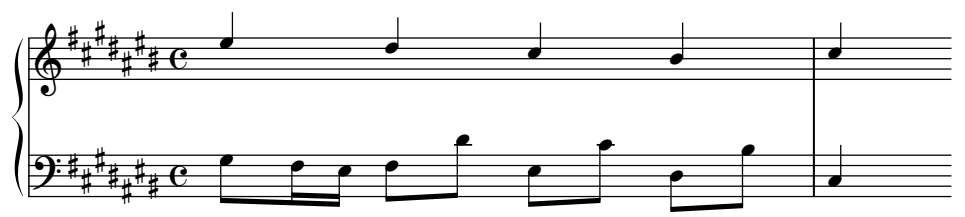

Beispiel 15: Thema im Bass (zweiter Takt), Sextparallelen in der Oberstimme

Wieder bietet sich für die Dreistimmigkeit der Fauxbourdonsatz an. Den Sextsprung des Themas im Bass kann die Mittelstimme auffangen, indem sie jeweils terzweise abwärts geht (Beispiel 16).

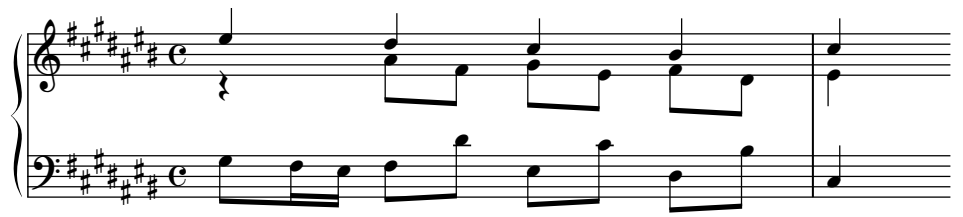

Beispiel 16: Thema im Bass (zweiter Takt), dreistimmig gesetzt

Indem wir die Mittelstimme diminuieren und die Oberstimme durch Vorhalte anreichern, gelangen wir auch hier wieder direkt zum dreistimmigen Schluss der Exposition in Bachs Fuge (Beispiel 17).

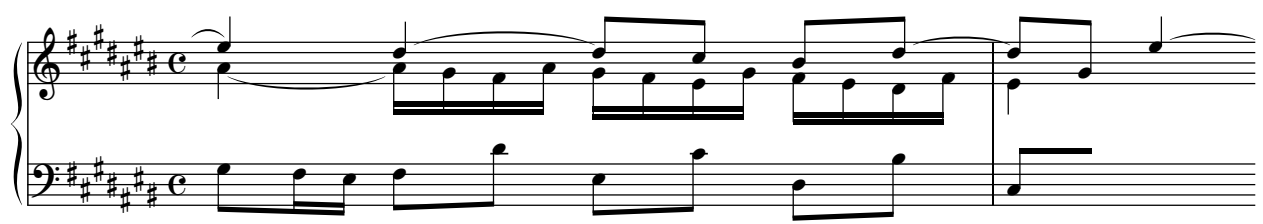

Beispiel 17: Johann Sebastian Bach, Wohltemperiertes Klavier (1722), Fuge Cis-Dur BWV 848, T. 6-7

Wir haben nun an zwei Beispielen gesehen, dass unsere Überlegungen nicht nur zum Thema, sondern jeweils direkt zu jener dreistimmigen Fassung führten, die bei Bach am Ende der Exposition steht. Wenn wir damit den Entwicklungsprozess zur Fugenexposition richtig herleiten, so ergeben sich zwei neue Thesen:

\section{These 3}

Im dreistimmigen Satz erfährt das Thema seine idealtypische Darstellung als Teil eines modellbasierten Stimmenkomplexes. So kann die Schlussdisposition für eine dreistimmige Exposition als `Kernstück $\imath^{19}$ formuliert werden. 


\section{These 4}

Die Exposition wird von ihrem Ende her komponiert. ${ }^{20}$

Anhand der Cis-Dur-Fuge lässt sich zudem gut zeigen, welche Konsequenzen eine entsprechende Themendisposition für die großräumige Anlage einer Fuge haben kann. Ist ein Thema sequenziell gebaut, bietet es eine Materialgrundlage für die Bildung sequenzieller Zwischenspiele. Aus dem Fauxbourdonsatz kann durch andere metrische Gliederung eine Terzfallsequenz werden (Beispiel 18).

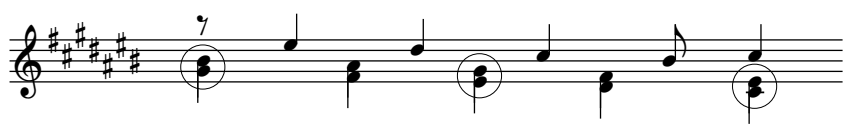

Beispiel 18: Zusammenhang zwischen 7-6-Kette, Fauxbourdon und Terzfall

Der Fauxbourdon kann durch Diminution der Bassstimme in eine Quintfallsequenz umgeformt werden (Beispiel 19).

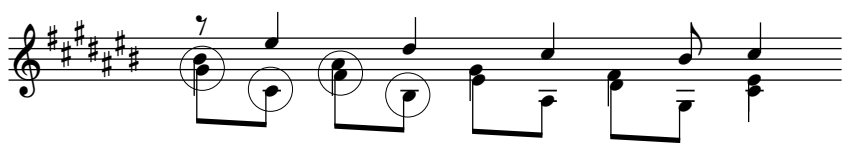

Beispiel 19: Zusammenhang zwischen Fauxbourdon und Quintfall

Bach bestreitet mit diesem Sequenzrepertoire die Zwischenspiele der Fuge: in einer Kombination aus Terz- und Quintfall (Beispiele 20 und 22), als terzweise sequenzierter Quintsprung abwärts mit gegenläufiger, terzweise ansteigender Stimme (Beispiel 21) und als Quintfall (Beispiel 23).

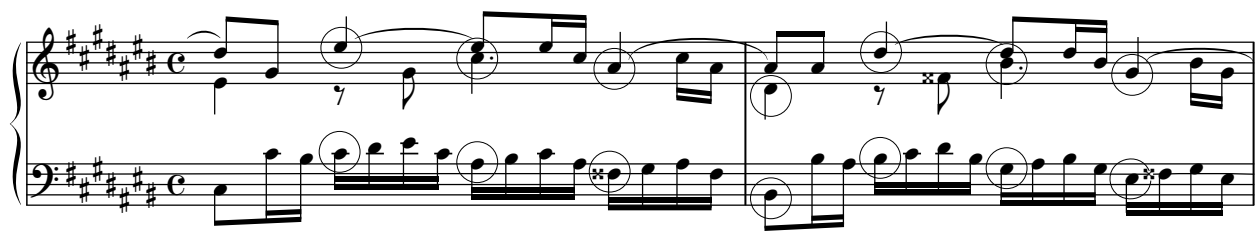

Beispiel 20: Johann Sebastian Bach, Wohltemperiertes Klavier (1722), Fuge Cis-Dur BWV 848, T. 7-8

19 Diesen Begriff führte Christoph Hohlfeld ein; vgl. Hohlfeld 2000, 24 (zur C-Dur-Fuge), 41 (zur c-Moll-Fuge) und 300 (Definition).

20 Die Thesen 3 und 4 legen es nahe, dreistimmige Fugen als Ausgangspunkt für den Unterricht zu wählen - denn hier muss der idealtypische Triosatz weder zur Vier- oder Fünfstimmigkeit ergänzt noch auf die Zweistimmigkeit reduziert werden. 


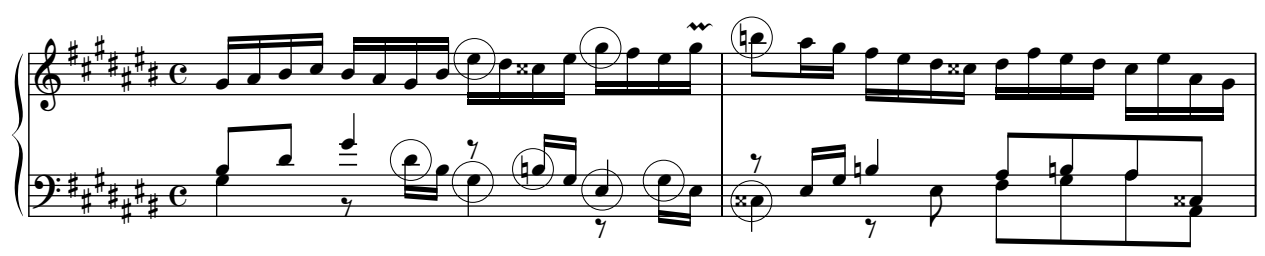

Beispiel 21: Johann Sebastian Bach, Wohltemperiertes Klavier (1722), Fuge Cis-Dur BWV 848, T. $12-13$

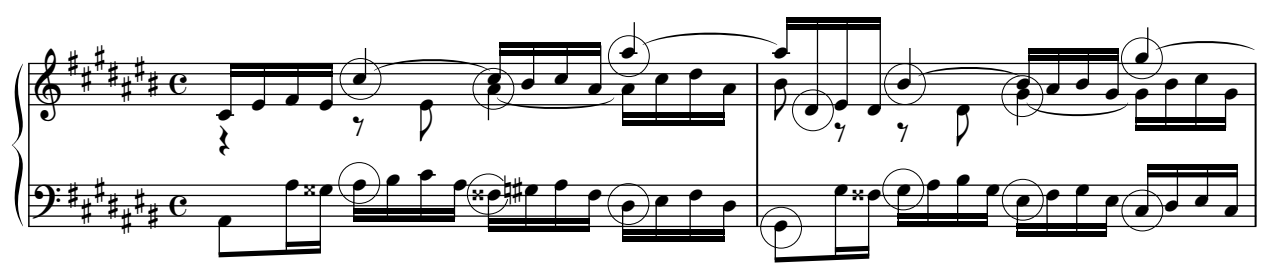

Beispiel 22: Johann Sebastian Bach, Wohltemperiertes Klavier (1722), Fuge Cis-Dur BWV 848, T. $16-17$

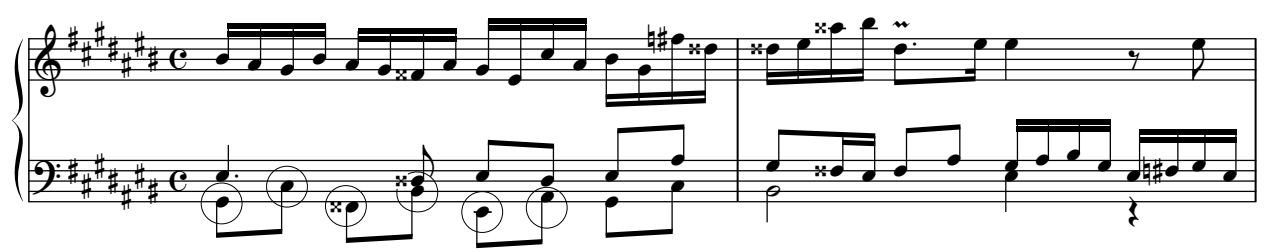

Beispiel 23: Johann Sebastian Bach, Wohltemperiertes Klavier (1722), Fuge Cis-Dur BWV 848, T. 21-22

Das Beispiel belegt, wie weitgehend das Thema auch die Zwischenspiele der Fuge bestimmen kann. ${ }^{21}$ So kommen wir zu einer weiteren These:

\section{These 5}

Das Thema kann darüber hinaus den Bau der Fuge beeinflussen: Wenn es sequenziell gebildet ist, sequenzielle Bildungen impliziert bzw. Sequenzbausteine zur Verfügung stellt, gibt es dem Komponisten Material an die Hand, das er in der Fuge entfalten kann.

\section{Das Thema aus einer Klausel-Wendung}

Das Thema der cis-Moll-Fuge (Beispiel 24) lässt sich als eine Kombination der beiden Primärklauseln fassen, zwischen denen das Thema sprungweise wechselt (Beispiel 25). 


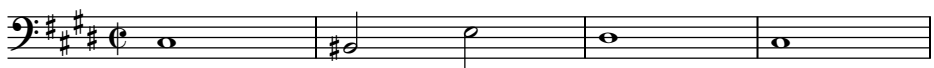

Beispiel 24: Johann Sebastian Bach, Wohltemperiertes Klavier (1722), Fuge cis-Moll BWV 849, Thema

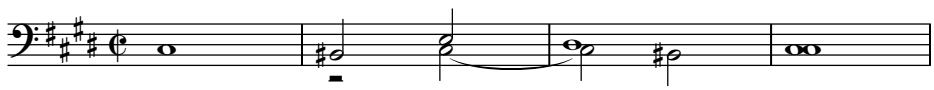

Beispiel 25: Johann Sebastian Bach, Wohltemperiertes Klavier (1722), Fuge cis-Moll BWV 849, Herleitung des Themas aus dem Klauselgerüst

Zum dreistimmigen Satz ergänzt dies eine Terzmixtur zur Tenorklausel, die bereits auf der ersten Zählzeit einsetzen kann²2 (Beispiel 26).

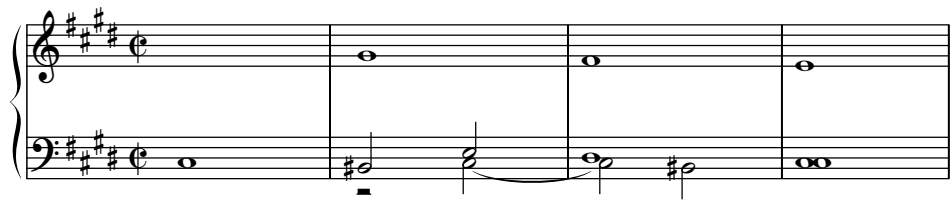

Beispiel 26: Erweiterung zum dreistimmigen Modell

Die Oberstimme erhält einen zusätzlichen Ansatztakt, und das Thema wird um eine Oktave nach unten versetzt, denn selbstverständlich sind die Primärklauseln vertauschbar (Beispiel 27). Es folgt die Diminution zunächst der Oberstimme (Beispiel 28) und dann der Mittelstimme, der Diskantklausel. Schließlich kann der Satz durch eine weitere Stimme zur Vierstimmigkeit ergänzt werden, so dass sich die Bachsche Konstellation von Thema und Kontrasubjekten ergibt (Beispiel 29).

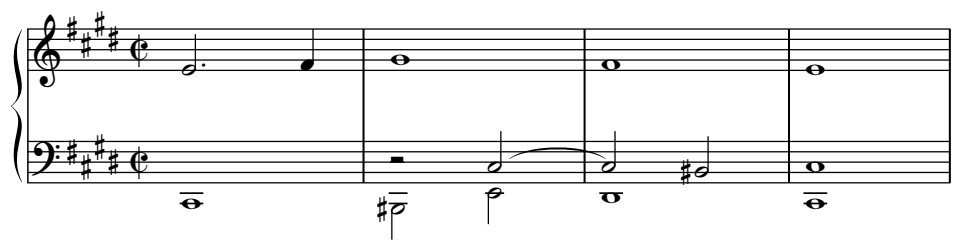

Beispiel 27: Dreistimmiges Modell plus Ansatztakt in der Oberstimme

21 Eine anders gelagerte großformale Konsequenz des Themas kann laut Christoph Hohlfeld in der Wiedergabe der »Konturtöne des Themas" als »Stationen« der Fuge liegen; er zeigt dies u. a. an der eingangs besprochenen C-Dur-Fuge (2000, 25).

22 Eine fast gleich lautende »inventio« formuliert Ariane Jeßulat $(2008,316 \mathrm{f}$.). 


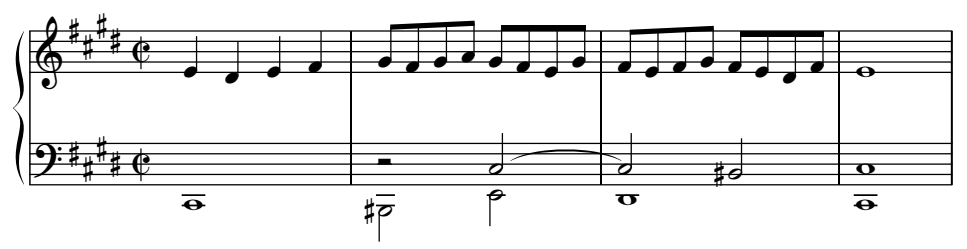

Beispiel 28: Diminution der Oberstimme

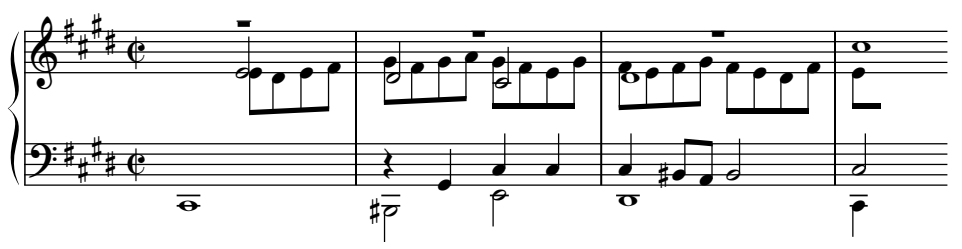

Beispiel 29: Johann Sebastian Bach, Wohltemperiertes Klavier (1722), Fuge cis-Moll BWV 849, T. 73-76

Aus den Überlegungen zur cis-Moll-Fuge folgt eine weitere Aussage:

\section{These 6}

Bei einer Doppel- oder Tripelfuge wird zunächst das Zusammentreffen der verschiedenen Themen festgelegt.

\section{Schlussfolgerungen}

Wie wir gesehen haben, lässt sich das Fugenthema als Extrakt eines modellbasierten Satzes verstehen. Bach wählt für jede der ersten vier Fugen des Wohltemperierten Klaviers einen anderen Ausgangspunkt: einstimmige Linie, Parallelismus, Vorhaltskette (bzw. Sequenz) sowie Klausel-Wendung.

Unser Versuch, den Weg zur Bachschen Themenbildung nachzuvollziehen, legt Konsequenzen für den Unterricht nahe:

- Insbesondere der Kerngedanke, dass am Anfang des Kompositionsprozesses einer Fuge nicht das Fugenthema steht, sondern eine satztechnische Idee, sollte im Unterricht reflektiert werden.

- In eigenen Übungen sollten Fugenthemen aus verschiedenen Elementarvorgängen (Linien, Parallelismen und Vorhaltsketten bzw. Sequenzmodellen) entwickelt werden, Figurationen improvisatorisch ausgeführt werden.

- Bildet aber ein fertiges Fugenthema den Ausgangspunkt einer Aufgabe, so beginnt die Komposition einer Fuge nicht bei Takt 1. Vielmehr wird das Thema zunächst im Hinblick auf seine kontrapunktischen Potentiale genau untersucht, im zweiten 
Schritt wird eine kompositorische Idee entwickelt und im dritten ein dreistimmiges 'Kernstückı für den Schluss der Exposition ausformuliert; hier zeigt sich das Thema in seinem modellgebundenen Zusammenhang.

- Auch Mischformen in Aufgabenstellungen sind praktikabel: Der Ansatz eines Fugenthemas kann gegeben sein, und das Thema ist so weiterzuführen, dass die Fuge in eine Richtung profiliert wird - etwa als Umkehrungs- oder Engführungsfuge.

Eine konkrete Aufgabenstellung könnte darin bestehen, ein Thema über ein Bassmodell zu bilden. Selbst der einfache Bassstufengang 1-4-5-1 kann hier erhellend sein (Beispiel 30):
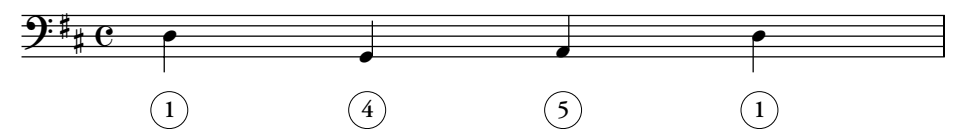

Beispiel 30: Bassstufengang als Fundament für ein Fugenthema

Eine schlichte Oberstimme hierzu könnte lauten (Beispiel 31):
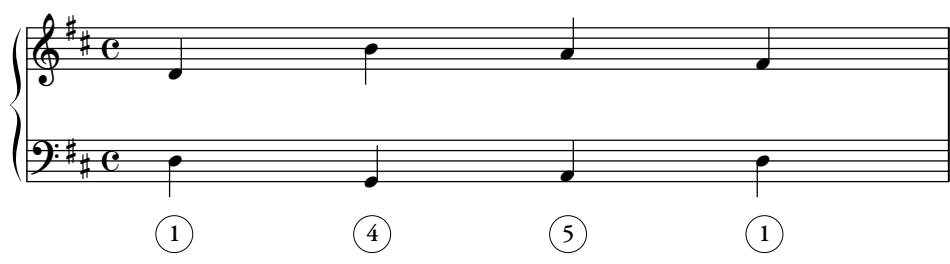

Beispiel 31: Oberstimme zum Bassstufengang

Nun fehlen noch Diminution und rhythmische Gestaltung - und schon sind wir, nach unserer Analyse der ersten vier Fugenthemen aus dem Wohltemperierten Klavier, beim Thema der fünften Fuge gelandet (Beispiel 32):

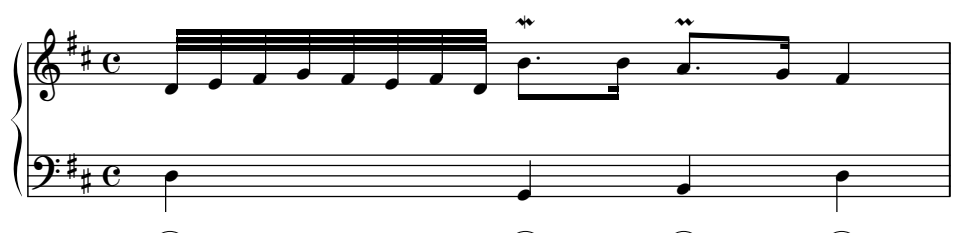

(1)

(4)

(5)

(1)

Beispiel 32: Johann Sebastian Bach, Wohltemperiertes Klavier (1722), Fuge D-Dur BWV 850, Thema mit unterlegtem Bass

Es zeigt sich: Das Lehrbuch Wohltemperiertes Klavier muss weiter gelesen werden. 


\section{Literatur}

Arnecke, Jörn (2002), »Idee, Umkehrung, Synthese. Das Thema der Fuge dis-Moll aus Johann Sebastian Bachs 'Wohltemperiertem Klavier«", in: Melodie und Harmonie. Festschrift für Christoph Hohlfeld zum 80. Geburtstag (= Musik und, Schriftenreihe der Hochschule für Musik und Theater Hamburg, Neue Folge 3), hg. von Reinhard Bahr. Berlin: Weidler, 85-103.

Dahlhaus, Carl (1989), Die Musiktheorie im 18. und 19. Jahrhundert, II. Teil: Deutschland (= Geschichte der Musiktheorie 11), Darmstadt: Wissenschaftliche Buchgesellschaft.

Deppert, Heinrich (2008), „Grundsätze zum Studium der Kompositionstechnik in den Fugen von J. S. Bach«, 1. Teil, ZGMTH 5/2-3, 287-312.

Froebe, Folker (2008a), Editorial, ZGMTH 5/2-3, 191-193.

(2008b), »Ein einfacher und geordneter Fortgang der Töne, dem verschiedene Fugen, Themen und Passagen zu entlocken sind . Der Begriff der sphantasia simplexı bei Mauritius Vogt und seine Bedeutung für die Fugentechnik um 1700«, ZGMTH 5/2-3, 195-247.

Grabner, Hermann (1934), Anleitung zur Fugenkomposition, Leipzig: Kistner \& Siegel.

Hinrichsen, Hans Joachim (2002), »Zur Wirkungsgeschichte des wohltemperierten Klaviers«, in: Siegbert Rampe (Hg.), Bach. Das Wohltemperierte Klavier I - Tradition, Entstehung, Funktion, Analyse. Ulrich Siegele zum 70. Geburtstag, München: Katzbichler, 27-51.

Hohlfeld, Christoph (2000), Johann Sebastian Bach, Das Wohltemperierte Klavier 1722. Schule musikalischen Denkens, Teil 2, Wilhelmshaven: Noetzel.

Jeßulat, Ariane (2008), „Nam et expectat et attendit et meminit - Dimensionalität von Zeit und Zeiterfahrung in der Fuge cis-Moll BWV 849«, ZGMTH 5/2-3, 313-333.

Marpurg, Friedrich Wilhelm (1753/54), Abhandlung von der Fuge [...], 2 Bde., Berlin, Reprint Hildesheim u. a.: Olms 1974.

Mattheson, Johann (1739), Der vollkommene Capellmeister, Hamburg, Reprint, 5. Aufl., Kassel: Bärenreiter 1991 (= Documenta Musicologica, Druckschriften-Faksimiles 5).

Polth, Michael (2008), »Satztechnische und strukturelle Stimmführung im frühen 18. Jahrhundert. Zur Bedeutung des Fugensoggettos für den musikalischen Zusammenhang", ZGMTH 5/2-3, 249-286.

Preuß, Volkhardt (2002), »Die Fuge zwischen Rezeption und Wandel«, in: Melodie und Harmonie. Festschrift für Christoph Hohlfeld zum 80. Geburtstag (= Musik und, Schriftenreihe der Hochschule für Musik und Theater Hamburg, Neue Folge 3), hg. von Reinhard Bahr, Berlin: Weidler, 63-84.

Richter, Ernst Friedrich (1886), Lehrbuch der Fuge, Leipzig: Breitkopf \& Härtel.

Schmoll-Barthel, Jutta (2002), „Wege eine Fuge Bachs zu verstehen«, in: Siegbert Rampe (Hg.), Bach. Das Wohltemperierte Klavier I - Tradition, Entstehung, Funktion, Analyse. Ulrich Siegele zum 70. Geburtstag, München: Katzbichler, 235-320.

Sprick, Jan Philipp (2008), »Fugen-Lehrbücher im 20. Jahrhundert«, ZGMTH 5/2-3, 347370. 